\title{
Food Allergy and Breastfeeding
}

\author{
Arnaldo Cantani* \\ Pediatric Clinic, University "La Sapienza" Roma, Italy
}

Submission: January 19, 2017; Published: January 23, 2017

*Corresponding author: Arnaldo Cantani, Pediatric Clinic, University “La Sapienza” Roma, Italy

Abbreviations: BM: Breast milk; SPF: soy protein formulas; HFs: hydrolysate formulas; EFAs: essential fatty acids; AD: atopic dermatitis; GJA: gamma-linolenic acid; CMA: CM allergy; PUFA: polyunsaturated; LCP: long chain; AA: arachidonic; M-CSF: macrophage colony-stimulating factor; BLG: ß-lactoglobulin

\section{Mini Review}

Allergy and Clinical Immunology Division, Department of Pediatrics, University of Roma "La Sapienza" Medical School Breastfeeding Normal neonates are equipped with a limited immunocompetence, therefore they need breast milk (BM), which represent an excellent immune protection for the neonate during the critical period of intestinal vulnerability, due to a great variety of functionally interactive immunological, antibacterial, antiviral, anti-inflammatory and immunomodulating factors. Evidence suggests that the protection afforded by human milk to the recipient infant is greatest when breast-feeding is exclusive and of substantial duration. In this update of an old topic, we shall review its main role in atopy prevention as an introduction to the immunological and non immunological components of $\mathrm{BM}$ and colostrum, and the spectrum and mechanisms of the protection of host defenses.

Accordingly, we analyzed the propensity for breastfeeding in 289 children with respiratory disease and in 300 control children. The net result is that a high proportion of atopic children (273/289) were breastfed from their mothers and for a longer period of time. Conclusion this is the best demonstration that breastfeeding is the most effective single nutritional strategy that has been identified for the prevention of the atopic march in vulnerable infants. Therefore we stress that breast-feeding can prevent or ameliorate allergies, although some authors have emphasized the increased hazard of sensitization in breastfed infants. There is a continuous flow of studies stressing the effectiveness of (exclusive breastfeeding associated or not with soy protein formulas (SPF) and/or hydrolysate formulas (HFs), along with food and inhalant allergens avoidance) in decreasing the prevalence of allergic diseases in genetically at risk neonates.
The protective effects of breastfeeding are indeed a positive natural selection process. The allergy preventive BM importance has been evaluated by both prospective and retrospective studies. We have prepared a partial list of both types of studies, which show that the BM protective value of BM is confirmed by $37 / 41(90,3 \%)$ of prospective, and by $4 / 9(44,4 \%)$ retrospective studies. Therefore the studies showing either no differences or an increase of allergic disease in breastfed in comparison with the bottle-fed children total to $8 / 40$. As was pointed out in some cases the infants were breastfed for less than 3 months ( $47 \%$ of children in one study) sometimes less than 6 weeks. Solid foods were introduced very early in the infants' diet $75 \%$ of cases at the $4^{\text {th }}$ month in another study) with foreseeable effects e.g. the start of the atopic march. As regards the importance of environmental controls, as we proposed and applied together with dietary manipulations: these measures, demonstrating that they are highly effective in reducing the prevalence of allergic disease: $14 \%$ vs $40 \%$ according to the employment or not of environmental controls in babies, who were all fed either with $\mathrm{BM}$ or a soy HF.

The figures are quite similar according to the dietary manipulations (breast or SPF in the study group, cow's milk - CM - in the controls) as $\neg$ sociated with the environmental controls. The results showed that 51/244 children developed atopic symptoms during the follow-up: when we consider together the study group, only $14,5 \%(26 / 179)$ compared to 38.5 (25/65) of the control group developed atopic manifestations during the follow-up thus confirming that dietary and environmental measures are able to significantly prevent the atopy onset in high-risk babies, at least until the age of 3 years and 8 months. 
Simi-lar results we obtained in a multicenter study. At the last follow-up 732 children are 3 years old, 98 (13\%) have two or more relatives, $634(87 \%)$ have one relative affected by atopic disease. $242 / 732$ (33\%) were exclusively breast feed until the 6th month of life, 139/732 (19\%) received exclusively SPF, 212/732 (29\%) received BM and SPF supplement, and 139/732 (19\%) were CM formula feed.

The prevalence of atopic disease was 13\% (77/593 of the study group vs. $48 / 139=34.5 \%$ of the controls, in this prospective study in high-risk babies we have verified how many of them were breastfed, in comparison with the children of the control group. In order to explore how long a baby genetically at risk of atopy is breastfed, we have enrolled into these prospective study 289 children, 169 males and 120 females, aged 3.5 to 7.5 years. These children attended the Allergy and Clinical Immunology Division of the Pediatric Dept of Rome University, because they were affected by respiratory allergy, previously diagnosed with family history, SPTs and IgE antibodies. As controls there were 300 children comparable for age and sex with no respiratory illness recruited from our outpatient clinic. In particular we asked the accompanying parent(s) of the 589 children whether they were breastfed, and in case of positive response, which was the duration of breastfeeding.

The parents of all children gave their informed consent. Data were analyzed using the X2 method. Analysis males versus females $p=0.0001$. The mothers of the study group have breastfed $273 / 289$ off springs $(94,5 \%)$ of cases ( $p=0.0225)$ for 136 days (mean 125, range 33-211) $p=0.0001$. The control children were breastfed in $89,6 \%$ of cases, for a mean of 98 days (mean 80 days, range 10-110). In both groups breastfeeding was not always exclusive. The mothers of children at genetic risk were much more motivated, and have breastfed their children for a significantly longer period of time than the mothers of the control babies. The prospective preventive studies have largely confirmed the high value of breastfeeding alone or associated with dietary manipulations, along with environmental controls in the prevention of allergic disease in at risk babies. It was already stressed elsewhe $\neg$ re that postponing the atopy development leads to a lessening of the severity of the clinical manifestations, and even to atopy avoidance forever.

However, there are very sophisticated measures to cast doubt on the BM "completeness", such as indirectly throwing discredit on breastfeeding. There are some controversies and the reader can conclude that the above studies have yielded conflicting results, and that the ability of breastfeeding to delay the onset or to reduce the severity of allergic disease is only equivocal. For example Vandenplas affirmed that although the majority of studies suggest that exclusive breast feeding exert a protective effect, a number of other studies fails to show this assumption and point out that breastfeeding is not associated with a decrease in the subsequent risk of atopic disease. In addition, breastfeeding has been associated even with a rise in prevalence of atopic diseases, and a possible interference with normal growth. There are further, direct measures to comply this effect, such as advertising infant formulas in hospitals or the use of HFs in the Maternity Hospitals and even for atopy prevention in at risk infants.

In addition we refer to the necessity stressed by several authors of supplementing the diet of breastfeeding mothers with essential fatty acids (EFAs) and/or administering EFAs to children with atopic dermatitis (AD). Since 1929 we have learned that an EFA deficit may be the cause of skin lesions in animals and in atopic children compared with healthy controls. EFAs are polyunsaturated fatty acids that cannot be synthesized by vertebrates and must therefore be obtained from the diet. Dietary EFAs and their derived compounds are important structural components of cell membranes. They are the chief determinants of membrane fluidity and affect the activity of a number of membrane-associated enzymes. The eicosanoids play an important role in immunological and inflammatory processes both as effector signals and as immunoregulatory mediators. Increased LA proportions and decreased proportions of its derived fatty acids have been found in the plasma of AD patients.

Supplementation of the diet in these patients with gammalinolenic acid (GLA) in the form of evening primrose oil produces a tendency towards normal of the EFA (essential fatty acids) profile and an improvement in symptoms. Another possible drawback of BM resides in the so-called BM allergy, starting from the supplements of $\mathrm{CM}$ formulas occasionally given to full term healthy neonates in nurseries, even in breastfed babies. The amount of CM proteins in such supplements is enormous compared with the extremely low amount provided by BM. We know that $40 \mathrm{ml}$ of BM contains an amount of $ß$-lactoglobulin (ßLG) of $0,012 \mathrm{ng} / \mathrm{l}$, whereas $40 \mathrm{ml}$ of CM contains $1610 \mathrm{ng} / \mathrm{l}$ of ßLG! As a consequence of these occasional supplements, sensitization may occur in a predisposed baby, and the minute amount of CM proteins of BM may subsequ 7 ently act as a booster dose, triggering allergic reactions. The results of several studies support this hypothesis: CM allergy (CMA) was significantly more common in babies who received supplements of $\mathrm{CM}$ formulas early in life in comparison to fully breastfed babies.

All the fully breastfed babies who developed CMA received feedings of CM formula in the nurseries and none of the fully breast fed babies without supplements of CM formula developed CMA. Lindfors et al. have documented that children with skin prick tests (SPT) and specific IgE antibodies against egg all were fed CM during the first days of life. Total IgE levels at the 5 th day of life were significantly correlated with the amount of early postnatal CM supplementation ( $p=0.013)$, maintaining the significativity until the age of 12 months mainly in at risk babies. This data confirms the studies cited so far and the classic Jarrett one (the repeated little doses of allergens are more sensitizing than larger ones for the predisposed individual). In addition, $93 \%, 68 \%$, or $64 \%$ of breastfed infants were exposed 
to less or more inadvertent supplements in the neonatal nursery. The babies presented CMA proved by challenge on an average after 7 weeks of life, or immediate symptoms at the first CM introduction, at the age of 1-8 months (median 4.0).

We emphasize that when the "pirate bottle" has administered HFs: the neonates presented with anaphylactic reactions when they were fed such formulas on weaning from BM. The infants kept an immunologic memory of the type of supplement received at that time. Since several years in the Maternities of Northern countries and in a London hospital, these CM feedings are no more permitted. A typical case is reported by Lifschitz an anaphylactic shock due to CM protein hypersensitivity in a newborn who was mistakenly fed BM that had been expressed before CM products were eliminated from his mother's diet is correctly shown in the title, however in the abbreviated title and in the discussion is referred to as "anaphylactic shock in a breast-fed infant"

The anaphylactic reactions triggered in young infants by the first CM administration show that apparently it is not easy to protect neonates at risk of atopy. Such reactions could be explained by transfer of maternal antigens directed versus the antigen-binding site of anti-idiotypic antibodies: if anti-idiotype antibodies against poliovirus antigens can be transferred from the mother to the offspring, similarly anti-idiotype antibodies to food antigens (e.g. lactoprotein), having the capacity of recognizing an idiotope within the paratope, could replace the antigen, mimicking its functional properties, and be transferred from the mother via the placenta to the fetus, acting like antigens during the neonatal period or subsequently. Therefore IgEmediated sensitization through BM is rather rare: $0.042 \%$ or $0.28 \%$.

Immunology of breast milk: nutrition and defense. It is increasingly manifest that BM contains a wealth of immune factors, which are designed to nourish and protect the vulnerable newborns during the critical postpartum period. Thanks to the mammary gland, a true immune organ, BM represents an excellent protection against the dangers of a deficient intestinal defence system, provides, together with colostrum, in addition to $\mathrm{T}$ and $\mathrm{B}$ lymphocytes and Ig, IFN, complement compo $\neg$ nents and other bioactive molecules protecting against bacterial and viral infections, idiotype/anti-idiotype antibodies may have lasting effects on the offspring immune system, EFA, polyunsaturated (PUFA), long chain (LCP) C20 and C22: arachidonic (AA, 20:4w6), docosahexaenoic (22:6w3), dihomo-g-linolenic (DGLA 20:3w6) necessary also for intellectual development. nucleotides, monomeric units of polymeric DNA and RNA, present in BM (\% of nitric products) five times more than in CM are essential in energy metabolism, enzymatic reactions, and during rapid growth, several factors insure a nonspecific protection against potential pathogens, leukocytes consist of (\%): neutrophils 55-60, macrophages 35-40, lymphocytes 5, 80\% of which are $\mathrm{T}$ lymphocytes, including memory $\mathrm{T}$ cells, macrophages are functionally active by means of M-CSF (macrophage colonystimulating factor) present with levels 10-100 times above the levels found in human blood (mean 30 times, ready to bridge the early neonatal "deficits".

Some oligosaccharides contribute to augment the defense potential of babies against infectious agents, acting as re $\neg$ ceptors for E. coli and Vibrio cholerae, preventing their adhesion to the intestinal mucosa, so decreasing the inflammatory reactions at the mucosal level. Immunology of colostrums centuries were necessary in order that its alimentary value was acknowledged, the immunologic one was still denied in 1939. Contains: IgA, IgM, IgG; as a compensation of poor quantity of IgG (3\% of maternal IgGs), colostrum and BM contain a significant concentration of subclasses, 》 $50 \%$ of maternal titres, sIgA, macrophages and EGF with titres higher than the BM, IgA antibodies (5-10 g/l) are transferred to newborns during the first 3 days after delivery: IgA can represent up to $80 \%$ of total content of proteins, nucleotide levels not much lower than those of transitional BM, factors binding $\operatorname{IgE}$ and specifically suppressing $\operatorname{IgE}$ synthesis by B cells of atopic individuals, factors eliciting a non-speci $\neg$ fic protective function at the level of mucosal barrier because the existent antibodies, not being absorbed, stick to the intestinal wall.

In addition an amount of EFA equal to the recommended ones, titres of CD mostly expressing IFN-g which assume a highly positive significance in the context of the above allu $\neg$ ded to inadequacy of such neonatal cells); moreover there is a double volume of TcR gd cells compared with that in the peripheral blood substances able to accelerate the development of an intact mucosal barrier, enhance the production of brush-border enzymes, and decrease food antigen penetration through an anti-inflammatory activity within the intestinal mucosa, antioxidant substances which could be feasible to inactivate $\mathrm{O}_{2}$ toxic metabolites, . colostral B cells respond with Ig secretion to the antigen stimulation; similar growth has been observed in culture. As far as we have as yet discussed it seems antiscientific and antimedical depriving the neonate of colostrum and early milk in the very first days of extrauterine life adds to the risk that potential pathogens from other foods and fluids may cause infections.

All evidence as yet gathered tends to prove that maternal breast is an immune organ belonging to MALT. BM not only has components protecting the vulnerable infant against the first infective and inflammatory episodes, but is also the vehicle for the transfer of immune regulation from the mother to her offspring, thus contributing to the maturation of the immune system of the newborn infant. Several immunomodulating factors present in BM that may actively modulate the baby immunologic growth, many of which are produced and are common to other mucosal sites, often share synergical features, provide a protective activity without inducing inflammation, moreover their production decreases with reference to the 
duration of breastfeeding and synchronously with the increased secretion of those factors from the neonate.

In addition EGF has been shown to play a role in reducing macromolecular absorption and to promote the functional maturation of the epithelial cells of the gut barrier, indeed the proliferation of the intestinal epithelium is more rapid in breastfed animals compared to the artificially fed ones SIgA is the major BM antibody, primed IgA-B cells home to the mammary gland through the enteromammary axis and are transferred to the suckling newborn, where they act against noxious intraluminal antigens and also respiratory microorganisms. In the neonatal gut were detected specific sites where maternal sIgA antibodies bind the glycocalyx of epithelial cells more firmly than the endogenous ones. Furthermore, maternal IgA antibodies have been shown to block effectively the antigen entrance into BM. Clearly, maternal MALT is in turn "educated" and, again through the enteromammary pathway, contributes to the de novo synthesis of sIgA; next as previously alluded to, the studies on anti-idiotypic antibodies show that they are favored in infants by maternal antibodies.

Even human milk macrophages play a role in the local protection of the infantile gut. Beginning from the first week of breast feeding, the baby receives spermine and spermidine, with a virtual protective effect on food allergy. Consequently it appears to be pivotal the immune defense insured to the offspring firstly by colostrum and then by BM, in a particularly critical period concerning a possible sensitization, in which the maturation of the gastrointestinal barrier and the antibody secretion still are inadequate. In conclusion, breastfeeding can prevent atopy development in genetically at risk newborns/ infants and promotes the maturation of the gastrointestinal tract with several mechanisms) however there are conditions potentially associated with immature mucosal barrier, for instance the elimination of gut flora following an antibiotic treatment. It is not out of place to state that the most common immunodeficiency may be that of the young infant deprived of BM with the ensuing paucity of $\operatorname{sigA}$ and other immune and defense factors (178). This is demonstrated by the study of 24 variables, as presumed causes of neonatal septicemia: only the protection insured by BM versus CM or formula reached the statistical significativity.

As regards possible effects of malnutrition on breastfeeding, neither the nutritional status, nor the ethnic origin influence the immunological components; instead Rotavirus infections may cause a significant rise in intestinal permeability to antigenic macromolecules (ßLG), in particular if associated with malnutrition, so that $\mathrm{CM}$ feeding can trigger an inflammatory reaction if the local microflora is re duced or absent. The development of sensitization to food antigens depends on genetic factors, however the phenotypic expression of the disease is modulated by the age of the baby or child and by the different diets administered early in life. Early exposure to food allergens in infancy is associated with an increased risk of sensitization, favoring the start of the atopic march. Exclusive, prolonged breast feeding and delayed weaning should be encouraged in babies of atopic families. Elimination of the most common offending foods from the maternal diet should be considered during the period of lactation.

There is no agreement on the most suitable formula if the mother cannot breastfeed. SPFs have been employed for many years and their safety and nutritional adequacy has been partly documented. In the last few years HFs have been suggested by several studies. However, at present, there are no data on the safety and nutritional adequacy on vulnerable babies exclusively fed these products since birth and for many months. Finally, due to the significant amount of immune reactive epitopes and intact CM proteins, HFs may be immuno genic in a predisposed host and should therefore not be given as a BM substitute further studies are necessary in order to rule out this likelihood before widely using these products in atopic prone babies. Only BM can prevent the atopic march, as confirmed by recent studies. This is best demonstrated by the high proportion of atopic children breastfed from their mothers in this study $=89,6 \%$.

The propaganda favor the diffusion of breastfeeding collides with the economical interests of manufacturers of cow milk and appropriate for atopic-allergic babies. Lactating mothers must avoid taking coffee and marijuana and similar beverages. Mothers taking the above drinks must procrastinate breastfeeding during the next during the next two hours, In addition to containing all the vitamins, hormones and nutrients the baby needs in the first six months of life, breast milk is packed with disease-fighting substances that protect your baby from illness. Every woman's journey to motherhood is different, but one of the first decisions a new mom makes is how to feed her child. When mothers choose to breastfeed, they make an investment in their baby's future. Breastfeeding allows mothers to make the food that is perfect for their baby. Their milk gives the baby the healthy start that will last a lifetime, Breastfeeding is therefore necessary to each neonate and maternal vicinity, she takes the baby among her arms, and this increases the reciprocal love.

Breast milk helps keep your baby healthy.
a. It supplies all the necessary nutrients in the proper proportions.
b. It protects against allergies, sickness, and obesity.
c. It protects against diseases, like diabetes and cancer.
d. It protects against infections, like ear infections.
e. It is easily digested - no constipation, diarrhea or upset stomach.
f. Babies have healthier weights as they grow.
g. Breastfed babies score higher on IQ tests. 
Breast milk is always ready and good for the environment.

a) It is available wherever and whenever the baby needs it.

b) It is always at the right temperature, clean and free. c) No bottles to clean.

d) Breastfeeding has no waste, so it is good for the environment.

e) Breastfeeding is free, has no concurrence

\section{Your next submission with Juniper Publishers} will reach you the below assets

- Quality Editorial service

- Swift Peer Review

- Reprints availability

- E-prints Service

- Manuscript Podcast for convenient understanding

- Global attainment for your research

- Manuscript accessibility in different formats ( Pdf, E-pub, Full Text, Audio)

- Unceasing customer service

Track the below URL for one-step submission https://juniperpublishers.com/online-submission.php 